\title{
Current Density Imaging and Electrically Induced Skin Burns Under Surface Electrodes
}

\author{
Andrei Patriciu*, Student Member, IEEE, Ken Yoshida, Member, IEEE, Johannes J. Struijk, \\ Tim P. DeMonte, Member, IEEE, Michael L. G. Joy, Member, IEEE, and Hans Stødkilde-Jørgensen
}

\begin{abstract}
The origin of electrical burns under gel-type surface electrodes is a controversial topic that is not well understood. To investigate the phenomenon, we have developed an excised porcine skin-gel model, and used low-frequency current density imaging (LFCDI) to determine the current density (CD) distribution through the skin before and after burns were induced by application of electrical current $(200 \mathrm{~Hz}, 70 \%$ duty cycle, $20-35 \mathrm{~mA}$ monophasic square waveform applied to the electrodes for 30-135 min). The regions of increased CD correlate well with the gross morphological changes (burns) observed. The measurement is sensitive enough to show regions of high current densities in the pre-burn skin, that correlate with areas were burn welts were produced, thus predicting areas where burns are likely to occur. Statistics performed on $\mathbf{2 8}$ skin patches revealed a charge dependency of the burn areas and a relatively uniform distribution. The results do not support a thermal origin of the burns but rather electro-chemical mechanisms. We found a statistically significant difference between burn area coverage during anodic and cathodic experiments.
\end{abstract}

Index Terms-Burn, current density, LFCDI, skin, surface electrodes.

\section{INTRODUCTION}

$\mathbf{S}$ URFACE electrodes are the most common electrodes used clinically, in applications as diverse as functional electrical stimulation (FES) for the restoration of motor function, pain relief, transcutaneous electrical nerve stimulation (TENS), electrocardiographic monitoring, defibrillation, surface cardiac pacing, and advanced drug delivery systems. Common to these applications are occasional reports of rash or burns on the skin at the points where electrodes were placed. 26 clinical cases of lowvoltage-induced burns are reported within [1]-[13]. More than half of these papers [1]-[7] report burns from surface electrodes, affecting nine patients. The origin of these electrical burns is not well understood, but the main theories are as follows.

- Oxygen and hydrogen gas bubbles, generated within the tissue during current passage, disrupt the stratum corneum

Manuscript received December 15, 2003; revised September 12, 2004. This work was supported by the Danish Academy of Technical Sciences. Asterisk indicates corresponding author.

*A. Patriciu is with the Center for Sensory-Motor Interaction, Aalborg University, DK-9220 Aalborg, Denmark (e-mail: ap@smi.auc.dk).

K. Yoshida and J. J. Struijk are with the Center for Sensory-Motor Interaction, Aalborg University, DK-9220 Aalborg, Denmark.

T. P. DeMonte and M. L. G. Joy are with the Institute of Biomaterials and Biomedical Engineering, University of Toronto, Toronto, ON M5S 3G9, Canada.

H. Stødkilde-Jørgensen is with the MR Research Center, Aarhus University Hospital, DK-8000 Aarhus, Denmark.

Digital Object Identifier 10.1109/TBME.2005.857677 creating regions of increased current density (CD) [14], [15].

- $\mathrm{pH}$ changes in the tissue under the electrode surface (decrease under anode and increase under cathode) [2], [16]-[18] suggest a chemical mechanism where alkali and acidic free radicals destroy the insulating epidermis, locally reducing the skin impedance.

- A thermal origin was also investigated [6], [16], [19], [20] but results are controversial regarding the magnitude of temperature rise under the electrode. Cellular damage induced by dissipation might occur within the skin appendages (sweat glands and hair follicles) [21] due to increased local CD.

A common factor contributing to all the abovementioned mechanisms are regions of high CD. A more evenly distributed current pattern through optimized electrode design achieved by a better understanding of the $\mathrm{CD}$ distribution could reduce the occurrence of burns during electrical stimulation.

Various methods were used to visualize these current paths: vibrating probe electrodes [22], electrode vehicle with an array of electrodes [23], special electrodes [24], scanning electrochemical microscopy (SECM) [25] or direct imaging techniques like scanning confocal fluorescence microscopy [26] or iontophoretic transport of tracer dyes [21], [27]. In vivo determination of current flow becomes difficult if not impossible for most of the mentioned techniques. Joy and co-workers [28]-[30] have demonstrated that low-frequency current density imaging (LFCDI), a magnetic resonance imaging (MRI) technique, is able to visualize the current flow path in vivo as well as in vitro experiments. The spatial resolution in their measurements was $0.78 \times 0.78 \mathrm{~mm}$.

In this paper, we report on work using the LFCDI technique [29], [31], [32] to determine the current distribution across the skin surface under a stimulating electrode before and after the burns were induced. To investigate the genesis of burns, we have developed an excised porcine skin-gel model and use the LFCDI to measure the CD distribution [33]. Numerous investigations have shown similarities between human and pig skin in terms of histology and histochemistry [34], ultrastructure [35], [36], or permeability to different chemicals [37], [38]. Despite existent differences, (thicker stratum corneum, less vascularity of the dermis and the lack of eccrine sweat glands) pigs have been used as animal models for the experimental and histological assessment of low-voltage electrical burns [39], [40], wound healing [41] as well as for the study of victims of electric torture in forensic sciences [42], [43]. 


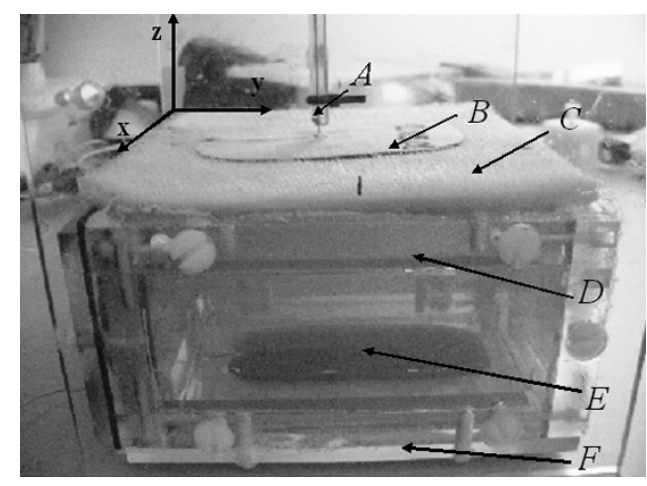

Fig. 1. Experimental skin-tissue model used in this study. The skin patch (C) is placed on the gelatin slab (D) mimicking the underlying tissue contained in the Plexiglas box $(\mathrm{F})$. The working electrode $(\mathrm{B})$ is connected via attachment (A). The electrical circuit is closed by the return electrode (E).

\section{METHODS}

LFCDI is a technique [31], which allows the direct visualization of volumetric current flow within an object, or within the body. According to Ampere's law

$$
\mathbf{J}=\mu_{0}^{-1} \nabla \times \mathbf{B}
$$

where

J CD vector $\left(\mathrm{A} / \mathrm{m}^{2}\right)$;

$\mu_{0} \quad$ magnetic permeability;

B magnetic flux density vector;

it is possible to determine the $\mathrm{CD}$ from a known magnetic flux density. The technique makes use of the phase information in the magnetic resonance (MR) image. The object is imaged while it is being actively electrically stimulated with current pulses. This imaging process is repeated in two orthogonal directions. This requires the object being measured to be small enough to fit within the bore of the MRI magnet. The only assumption is that the object can be imaged by the MRI technique.

$\mathrm{CD}$ images are calculated from the phase images recorded with the MR scanner. Detailed information about this algorithm can be found in [31], [32]. Briefly, the CD component normal to the surface of the skin patch, $J_{z}$, is computed using the partial Cartesian expansion of (1).

$$
J_{z}=\frac{1}{\mu_{0}}\left[\frac{\partial B_{y}}{\partial x}-\frac{\partial B_{x}}{\partial y}\right]
$$

where $\left(\partial B_{y} / \partial x\right)$ and $\left(\partial B_{x} / \partial y\right)$ are the spatial derivatives of the magnetic induction components with respect to $\mathrm{x}$ and $\mathrm{y}$ (Fig. 1).

The two orthogonal components of the magnetic field $\mathrm{B}_{\mathrm{x}}, \mathrm{B}_{\mathrm{y}}$ are determined by rotating the current conducting object by $90^{\circ}$ in the scanner. Derivatives of the induction components result by spatial filtering the unwrapped and scaled phase images [32].

\section{A. General Method}

An experimental skin-tissue model consisted of a Plexiglas box $(15 \times 14.5 \times 7 \mathrm{~cm})$ containing a homogeneous gelatine slab with a piece of porcine skin covering the top surface (Fig. 1). Similar to [33] and [44] the conductive gel was prepared using 1.51 distilled water, $200 \mathrm{~g}$ gelatine (MERCK Eurolab) and $3.37 \mathrm{~g} \mathrm{NaCl} .13 .6 \mathrm{ml}$ of formaldehyde was added as a preservative. The resulting homogenous gel has a conductivity of

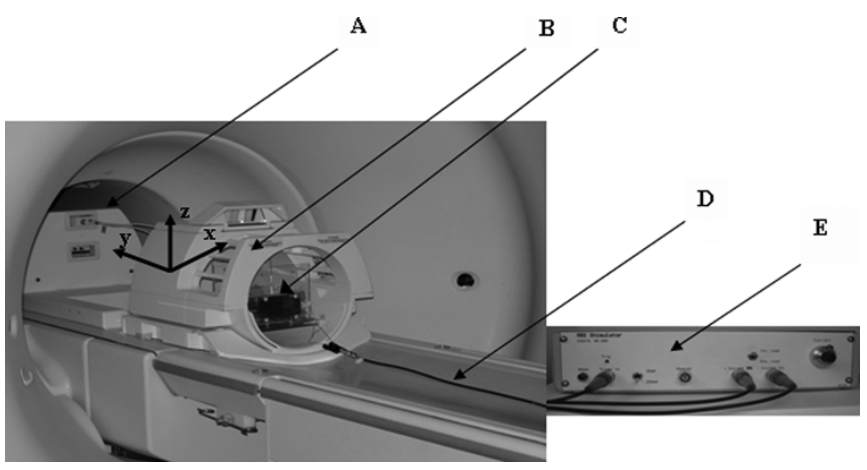

Fig. 2. The skin-tissue model (C) was placed into the head coil (B) and imaged inside the bore of a MR scanner (A) while connected by a pair of surface electrodes and connecting cable (D) to a specially designed LFCDI pulse stimulator (E). The imaging pulses were triggered by the MR sequence.

approx. $0.74 \mathrm{~S} / \mathrm{m}$ and mimics the electrical conductivity of the subcutaneous soft tissue.

We used skin collected from the lateral thigh surface of freshly euthanized Danish Landrace pig cadavers, $45-60 \mathrm{Kg}$ in weight. Prior to excision the skin surface was electrically shaved with care taken to minimize injury of the skin. Skin at this particular position has a relatively constant skin thickness $(1,5-2 \mathrm{~mm})$ with an approximately uniform subcutaneous fat layer giving a smooth inner surface. 28 rectangular patches of skin, $15 \times 14 \mathrm{~cm}$ in size, visually clear of scars and blemishes were collected for this study.

A pair of identical gel-type surface stimulating electrodes (Medicotest, Type 97-4031A, $50 \times 90 \mathrm{~mm}, 40 \mathrm{~cm}^{2}$ ) was applied to the top skin patch and the bottom side of the gel slab. The skin side electrode was always the working electrode while the gel side electrode was the counter electrode. The working electrode was prepared by cutting off the connecting lead wire and removing a part of the back cover to allow the soldering of an external, attachment wire [Fig. 1 and Fig. 3(A)]. This wire was positioned perpendicular to the surface of the electrode and used to connect the upper electrode in order to minimize LFCDI artefacts. The counter electrode and the gel slab were fixed to the Plexiglas box using a plastic lid. Both electrodes were connected to the LFCDI pulse generator. The top electrode was connected by soldering it to the wire attachment, while the bottom electrode was attached through its original lead wire and connector.

The skin-tissue model was placed on a plastic frame in the head coil of a General Electric Signa 1.5 T MRI scanner with the longitudinal axis of the working electrode aligned parallel to the main imaging field $\mathbf{B}_{0}$. The model was horizontally levelled so that MRI coronal imaging slices should be parallel to the skin surface and was connected to the $\mathrm{CD}$ imaging stimulator. Finally the Plexiglas box, the frame and the connecting wires were firmly fixed to the head coil. The setup used during the experiments is presented in Fig. 2. Each experiment was conducted following a three-step sequence:

\section{Pre burn imaging $\rightarrow 2$. Burn induction}

\section{$\rightarrow 3$. Post burn imaging}

in order to acquire photos of the skin surface and CD images of the skin-tissue model before and after inducing the burns. 


\section{B. Pre-Burn Imaging}

The CD component perpendicular to the imaged slice was determined using 5-50 mA bipolar symmetrical rectangular current pulses of 12-ms duration, synchronized with a spin echo MRI sequence. Special care was taken to charge balance the biphasic stimulation pulses to avoid burn-inducing processes during imaging. MRI parameters were: $\mathrm{TR}=2000 \mathrm{~ms}, \mathrm{TE}=$ $30 \mathrm{~ms}$, field of view (FOV) $26 \mathrm{~cm}, 256 \times 256$ recorded pixels, slice thickness $2 \mathrm{~mm}$ resulting in a voxel dimension of $1.02 \times$ $1.02 \times 2 \mathrm{~mm}$. A complete description of the multislice pulse sequence was described in [45]. Twenty-five slices with $0-\mathrm{mm}$ interslice gap were recorded in each imaging session. Prior to this a standard spin echo MRI sequence was run to locate the model on the magnitude image. This helped setting the scanner to start with the first slice at the surface of the working electrode. Since the acquired slices were "coronal" (in the xy plane as indicated in Fig. 2), and the surface electrode followed the shape of the porcine skin it was important during this experiment to keep the electrode-skin separation plane horizontal, meaning a constant skin thickness, and no air bubbles trapped between this and the gel slab. After the sequence was finished, the model was rotated $90^{\circ}$ counter clockwise around the $\mathrm{z}$ axis (Fig. 2) and re-imaged. These two measurements and the photographs of the skin comprised the pre-burn dataset. One complete imaging sequence took $35 \mathrm{~min}$. Only the normal component of the CD to the skin surface was imaged during all experiments.

\section{Burn Induction}

After the pre-burn data was collected, the model was removed from the scanner and burn-inducing stimulation was carried out using a $200 \mathrm{~Hz}, 70 \%$ duty cycle, 20-35 mA monophasic square waveform applied to the electrodes for 30-135 min time to make the charge $\mathrm{Q} \approx 45-115 \mathrm{C}$ using a stimulator (Grass, SD9) and a current isolator (Grass, PSIV6). 14 experiments were conducted with anodic stimulation related to the working electrodes, and 14 experiments were conducted with cathodic stimulation. These stimulation values were selected based on studies, which showed that these parameters cause burns in all trials. Burns were induced by maintaining an average $\mathrm{CD}$ higher than $0.5 \mathrm{~mA} / \mathrm{cm}^{2}$ but selecting a current in the range of 20-35 mA, which minimized the problems of gassing the electrode hydrogel and the possibility of heat induced burns. Studies have determined [16], [46], [47] that currents below $0.5 \mathrm{~mA} / \mathrm{cm}^{2}$ of the conductive electrode surface can be considered safe during iontophoretic treatment in human. The expected a priori area of the burns was not known for any of the trials.

\section{Post-Burn Imaging}

Following the burn induction, the phantom was placed in the MR imager again and using the same imaging parameters as during pre-burn imaging the post-burn $\mathrm{CD}$ distribution was recorded. When the sequence was finished, the top electrode was peeled off and photos of the exposed skin surface taken to document the experiment. These photos together with the post-burn MRI measurements comprised the post-burn data set.

\section{E. Image Processing and Analysis}

During each experiment photographs of the surface of the skin were taken using a digital camera (Fuji, FinePix 4700) to record its burn features before and after the procedure. Photos were taken with the camera mounted $20 \mathrm{~cm}$ above the skin-gel model at a resolution of $1289 \times 960$ pixels. Care was taken to have the camera lens parallel to the surface of the skin and centerd above it to minimize optical distortions.

The images of the skin before and after the induced burns were digitally processed using Matlab (The MathWorks Inc., Natick, MA) to compensate for the luminance variation across the picture due to the illumination source. Burns appear on these images as dark brown stains on the skin surface. Consequently, the burn images were converted to gray scale and thresholded using Paint Shop Pro (Jasc Software, Inc., Eden Prairie, MN) to identify and measure the area of skin with burns. Since the skin was initially free of blemishes or scars, only the burn pixels were marked as " 1 " compared to pixels of the unburned region, which were marked " 0 ." We computed the resulting burn area as the ratio of the number of " 1 " pixels ("burned") divided by the total number of pixels beneath the electrode. The corrected greyscale photos of the skin before and after the burn induction were downsampled to a picture of $49 \times 87$ pixels corresponding to the size of the electrode from the $\mathrm{CD}$ images. This allowed 1:1 comparison between photo pixels and CD values. Finally both image files were masked so that only the skin under the electrode was compared. Three separate $49 \times 87$ pixel binary masks were generated for each data set: one for the CD image before inducing the burn one for the CD image after and one for the skin images before and after inducing the burns. The CDI masks exhibit " 0 " where one of the following conditions is met: 1) negative $C D$ values due to artefacts (high phase gradients); 2) zero $C D$ values due to signal-to-noise ratio (SNR) thresholding (pixels blanked out by the $\mathrm{CD}$ computing routine); or 3) zero CD value due to zero current (regions outside the electrode boundary), and were set to " 1 " for the remaining "valid" pixels. The photo mask was set to " 0 " for the pixels outside the electrode boundary and " 1 " for the rest.

\section{F. Differences Between Anodic and Cathodic Burns}

The two samples of 14 anodic and 14 cathodic stimulations were compared using a Mann-Whitney U rank order test to look for a statistically significant difference. The statistical significance level was chosen to be $\alpha=0.05$ for all statistical tests in this study. Multiple regression analysis was further applied for a quantitative analysis. The investigated predictors were current, time, charge, current squared $\left(\mathrm{I}^{2}\right)$ and current squared multiplied by time $\left(\mathrm{I}^{2} \mathrm{t}\right)$. For each predictor we determined the multiple correlation coefficients $\mathrm{R}, \mathrm{R}^{2}$, the adjusted value $\mathrm{R}_{\mathrm{a}}^{2}$ and the significance level $\mathrm{p}$ [48].

\section{G. Correlations Between Burn Images and CDI}

For each experiment five correlation coefficients were computed, one for each of the following cases:

1) $\mathrm{CD}$ before versus $\mathrm{CD}$ after inducing the burn;

2)-5) CD before/after versus skin photo before/after inducing the burn (four combinations). 
CD images recorded after the burns were induced are referred in this paper as CDI post, while values recorded on the freshly excised skin as CDI pre. Consequently burn images are referred as photo post-as compared to the images of the initial skin (Photo pre). The correlation coefficients were calculated across the valid pixels resulting from the logical "and" function of the corresponding masks. The values were grouped in five categories denoted: CDI post-Photo post, CDI post-Photo pre, CDI pre-Photo post, CDI pre-Photo pre, and CDI post-CDI pre.

The resulting data sets were tested for normality within each group, and a one-way analysis of variance (ANOVA) test was performed. Significant results were further analyzed using the Tuckey HSD post-hoc test for multiple comparisons [48]. Values are presented as mean \pm standard deviation.

\section{H. Distribution of Burns}

In order to determine the randomness of the burn distribution across the skin surface, the data sets of 14 anodic and 14 cathodic binary burn images, processed as described in Section II-E and downsized to $49 \times 87$ pixels were separately summated. The resulting histograms were visually inspected.

\section{RESULTS}

A typical example of a burn inducing experiment is shown in Fig. 3. The photo of the skin surface [Fig. 3(C)] before placing the stimulation electrode [Fig. 3(A)] and the LFCDI of the same region [pre-burn imaging, Fig. 3(E)] are compared to the "post-burn" skin photo [Fig. 3(D)] and LFCDI [post-burn imaging, Fig. 3(F)] after peeling the electrode off. The MRI magnitude image through the surface electrode [Fig. 3(B)], suggests that the copper wire attachment is responsible for the artefacts around that region seen in the LFCDI images.

\section{A. Differences Between Anodic and Cathodic Burns}

We measured burn area coverage in the range of $3.1 \%$ to $54.6 \%$ of the total skin surface under the electrode in 28 data sets of induced burns. The 14 anodic values ranged from $3.1 \%$ to $14 \%(7.1 \pm 2.9)$ in contrast to a spread between $13.3 \%$ and $54.6 \%(31.1 \pm 11.0)$ for the 14 cathodic values. The MannWhitney-U rank order test showed a statistically significant difference between the two groups. $(\mathrm{p}<0.0001)$. Table I presents the results of the multiple regression analysis done on different subsets of the predictor variables charge (Q), current (I), and time (t) and separately $\mathrm{I}^{2}$ and $\mathrm{I}^{2} \mathrm{t}$.

For cathodic burns, the most significant dependency was related to charge $\left(\mathrm{p}=0.032\right.$ and $\left.\mathrm{R}_{\mathrm{a}}^{2}=0.272\right)$ followed by time $\left(\mathrm{p}=0.048\right.$ and $\mathrm{R}_{\mathrm{a}}^{2}=0.227$ ). The other predictor variables subsets were not statistically significant $(\mathrm{p}>0.05)$. Anodic burns expressed also a charge dependency $\left(\mathrm{p}=0.004, \mathrm{R}_{\mathrm{a}}^{2}=0.471\right)$ followed by the combination $t, Q\left(p=0.019, R_{a}^{2}=0.423\right) I, Q$ $\left(\mathrm{p}=0.0193, \mathrm{R}_{\mathrm{a}}^{2}=0.422\right)$ and $\mathrm{t}\left(\mathrm{p}=0.026, \mathrm{R}_{\mathrm{a}}^{2}=0.319\right)$. Neither data set showed significant dependency toward $\mathrm{I}^{2}$ and $\mathrm{I}^{2} \mathrm{t}$.

The linear regression of the burn area on charge for both anodic and cathodic data sets is presented in Fig. 4. As mentioned, the hypothesis that the slopes are zero was rejected for both regression lines. The slope for the anodic burns, $0.11(95 \%$

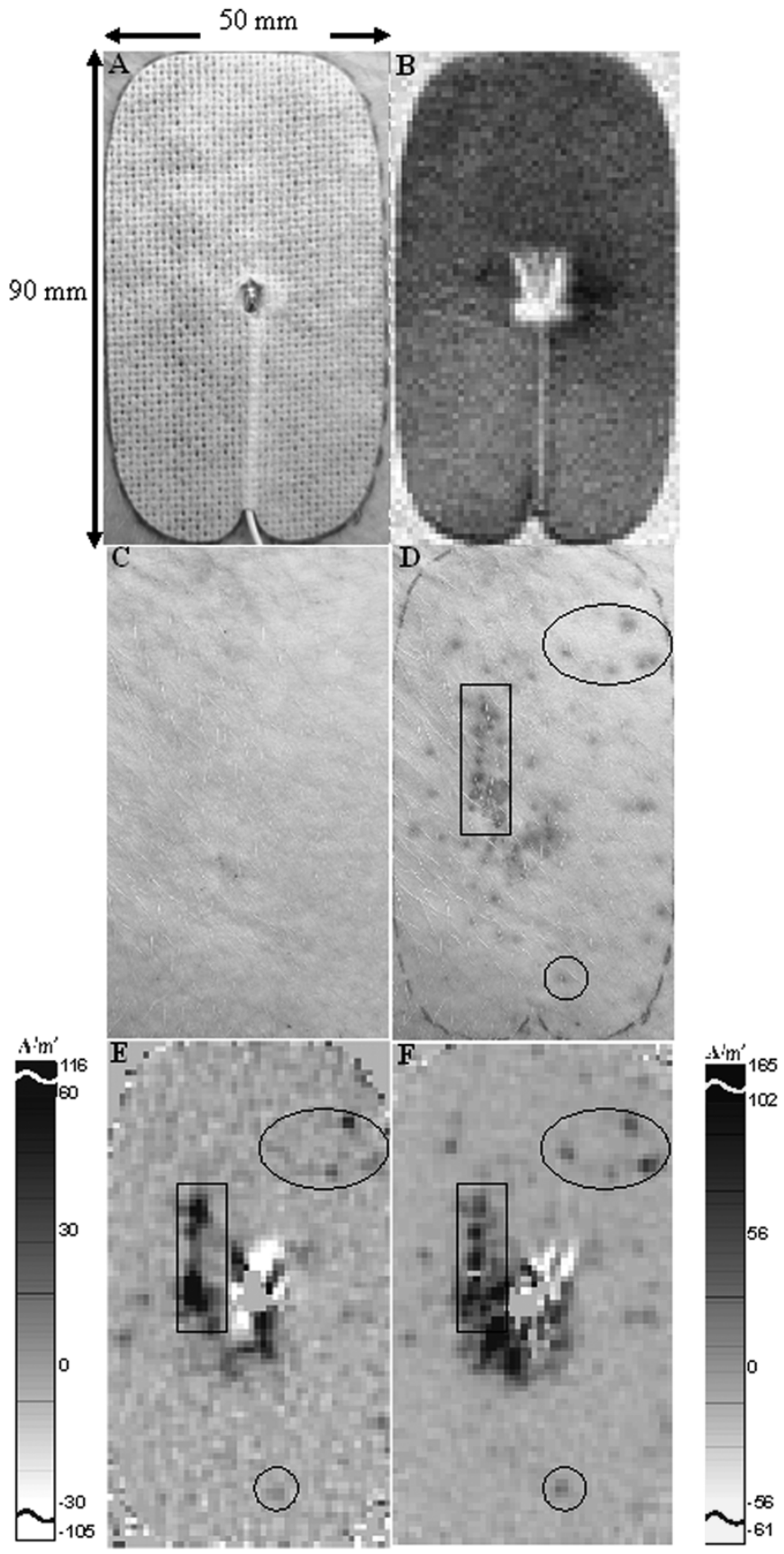

Fig. 3. Typical sequence of burn images. (A-top row left) Photograph of the electrode placed on the skin and the soldering place of the wire attachment Same location as seen in the MRI magnitude image, (B top row right), evidencing the copper wire and anchor in the middle of the electrode. A photo of the skin surface ( $\mathrm{C}$-second row left) before placing the electrode, and the same spot (D-second row right) after the burns were induced and the electrode peeled off. Dotted line shows the contour of the electrode. (C) and (D) Images before inducing the burn ( $\mathrm{E}$-bottom left) and after ( $\mathrm{F}$-bottom right). Note the CD scales attached to them. Some of the regions of interest were marked (circle, oval and rectangle) and examined in the corresponding images. The amplitude of the imaging current pulses is $15 \mathrm{~mA}$ in $(\mathrm{E})$, and $30 \mathrm{~mA}$ in $(\mathrm{F})$.

confidence interval 0.09-0.13) was found statistically different $(\mathrm{p}<0.003)$ from the slope of the cathodic burns, $0.41(95 \%$ confidence interval $0.34-0.48$ ). The intercept for both regression lines was assumed to be " 0 " knowing that there are no burns induced without current passage. 
TABLE I

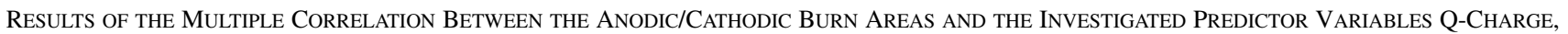

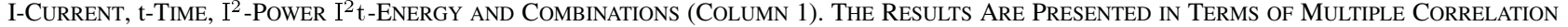
CoefFicient $(\mathrm{R})$, Adjusted VAlue of R $\left(\mathrm{R}_{\mathrm{a}}\right)$ And Sorted AcCording to Their Statistical Significance (p). Statistically not Significant PRedictors Were SEPARATEd Below the ACCEPTANCE Line $(\mathrm{p}<0.05)$

\begin{tabular}{|c|c|c|c|c|c|c|c|c|c|}
\hline \multicolumn{5}{|c|}{ Cathodic burns } & \multicolumn{5}{|c|}{ Anodic burns } \\
\hline Factor & $\mathrm{R}$ & $\mathrm{R}^{2}$ & $\mathrm{R}^{2} \mathbf{a}$ & $\mathbf{p}$ & Factor & $\mathrm{R}$ & $\mathrm{R}^{2}$ & $\mathrm{R}^{2} \mathrm{a}$ & $\mathbf{p}$ \\
\hline $\mathrm{Q}$ & 0.573 & 0.328 & 0.272 & 0.032 & $\mathrm{Q}$ & 0.715 & 0.511 & 0.471 & 0.004 \\
\hline $\mathrm{T}$ & 0.535 & 0.287 & 0.227 & 0.048 & $\mathbf{t}, \mathrm{Q}$ & 0.715 & 0.512 & 0.423 & 0.019 \\
\hline $\mathrm{I}, \mathrm{Q}$ & 0.581 & 0.338 & 0.218 & 0.103 & $\mathrm{I}, \mathrm{Q}$ & 0.715 & 0.512 & 0.423 & 0.019 \\
\hline $\mathbf{t}, \mathrm{Q}$ & 0.573 & 0.328 & 0.206 & 0.111 & $\mathbf{t}$ & 0.609 & 0.371 & 0.319 & 0.026 \\
\hline $\mathrm{t}, \mathrm{I}$ & 0.541 & 0.293 & 0.165 & 0.147 & t, I & 0.683 & 0.467 & 0.37 & 0.03 \\
\hline $\mathrm{Q}, \mathrm{I}, \mathrm{t}$ & 0.623 & 0.389 & 0.205 & 0.161 & $\mathrm{Q}, \mathrm{I}, \mathrm{t}$ & 0.716 & 0.513 & 0.367 & 0.056 \\
\hline $\mathrm{I}^{2} \mathrm{t}$ & 0.408 & 0.166 & 0.091 & 0.165 & $\mathrm{I}^{2} \mathrm{t}$ & 0.443 & 0.196 & 0.129 & 0.112 \\
\hline $\mathrm{I}^{2}$ & 0.277 & 0.077 & 0.0004 & 0.335 & $\mathrm{I}^{2}$ & 0.279 & 0.077 & 0.001 & 0.333 \\
\hline I & 0.273 & 0.075 & -0.002 & 0.343 & I & 0.258 & 0.67 & -0.1 & 0.371 \\
\hline
\end{tabular}

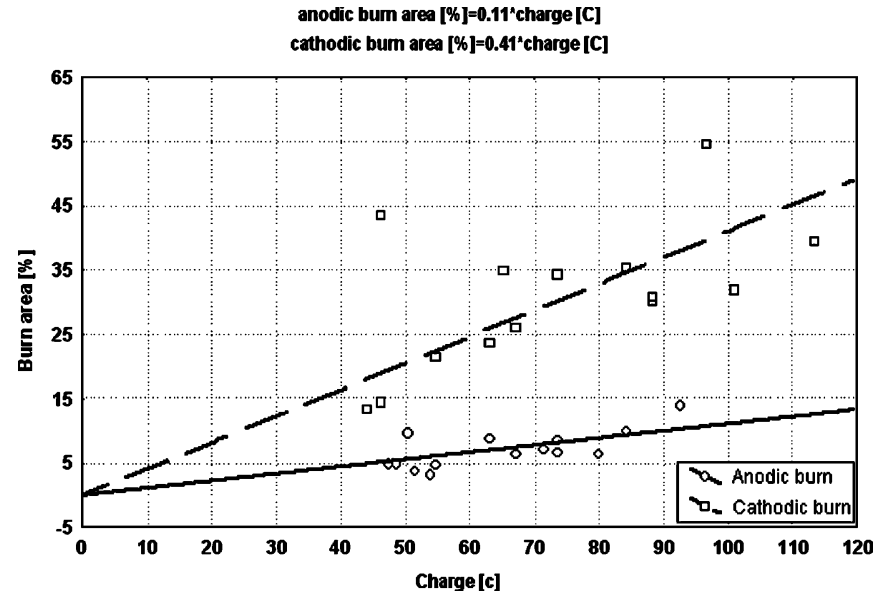

Fig. 4. Burn area versus charge during anodic and cathodic experiments. Multiple regression analysis performed upon the 28 burn inducing experiments revealed significant charge dependency of the recorded burns. The experimental data and the regression lines (dotted-cathodic burns, full-anodic ones) are shown in the figure. The anodic regression line has a slope of $0.11(0.092-0.13)$ and is significantly different $(\mathrm{p}<0.003)$ from the cathodic value $0.411(0.34-0.48)$. Values in brackets present the $95 \%$ confidence interval.

\section{B. Correlations Between Burn Images and CDI}

CD images were recorded in 18 out of the 28 burn experiments performed. The five groups of correlation coefficients computed from each data set are presented in Fig. 5. The one way analysis of variance shows significant differences between the mean values of these groups $(\mathrm{F} 4,85=38.6 ; \mathrm{p}<0.00001)$ The mean value of the correlation coefficients between CDI images and burn photos (CDI post--Photo post) was $0.31 \pm$ 0.17 . This value was significantly different from CDI post-Photo pre-(0.02 \pm 0.11$)$ as resulting from Tuckey HSD post-hoc test $(\mathrm{p}<0.00015)$. CDI pre-Photo post-correlations averaged 0.26 $\pm 0.16)$ and were found significantly different from CDI prePhoto pre- $0 \pm 0.12(\mathrm{p}<0.0002)$. The mean value of the correlation coefficients between CDI images, CDI pre-post-was $(0.49$ $\pm 0.17)$.

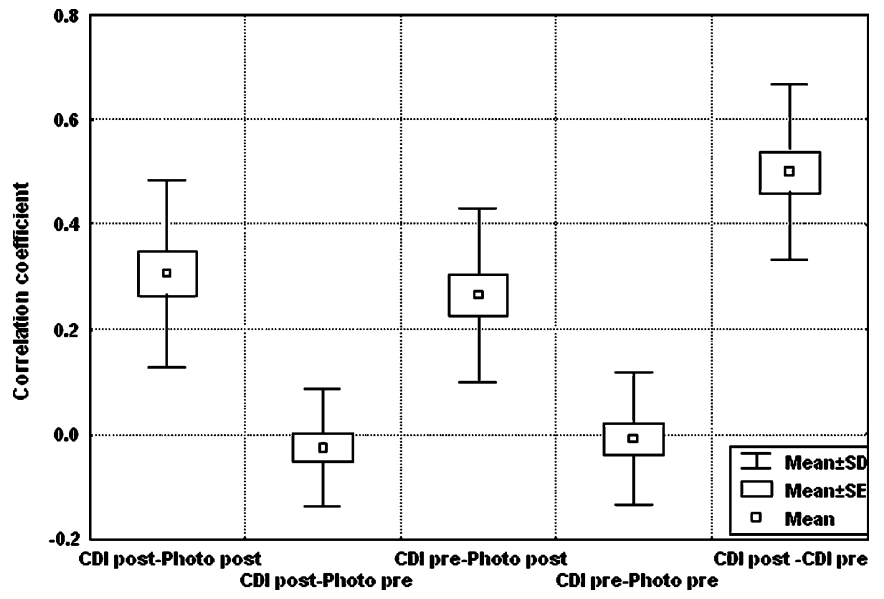

Fig. 5. Correlations between the photos of the skin and the CD images before and after inducing the burns. Values are representing the mean value \pm standard deviation for the following cases: 1 ) CDI post-photo post- $0.31 \pm 0.17$; 2) CDI post-photo pre- $0.02 \pm 0.11$; 3) CDI pre-photo post- $0.26 \pm 0.16$; 4) CDI pre-photo pre- $0.0 \pm 0.12$; and 5) CDI post-CDI pre- $0.49 \pm 0.17$. ANOVA analysis and Tuckey HSD post-hoc test revealed statistically significant $(\mathrm{p}<$ 0.05 ) differences between 1)-2) and 3)-4).

\section{Distribution of Burns}

Fig. 6 presents the distribution of the burns under the surface electrode separately summated for all anodic [Fig. 6(B)] and cathodic [Fig. 6(A)] experiments. On a scale from 0 (no burn detected) to 14 (region burned in all experiments) anodic burns ranged 0-6 as compared to cathodic ones ranging from 0 to 10 . A visual inspection of the two pictures shows a rather uniform distribution of burns across the skin area with no visible regions of burn clustering. A slight increase in the case of anodic burns can be observed in the region corresponding to the wire attachment [see, also, Fig. 3(B)].

\section{DISCUSSION}

One of the aims of the study was to relate CD measurements using LFCDI to anodic and cathodic burns. We chose to com- 


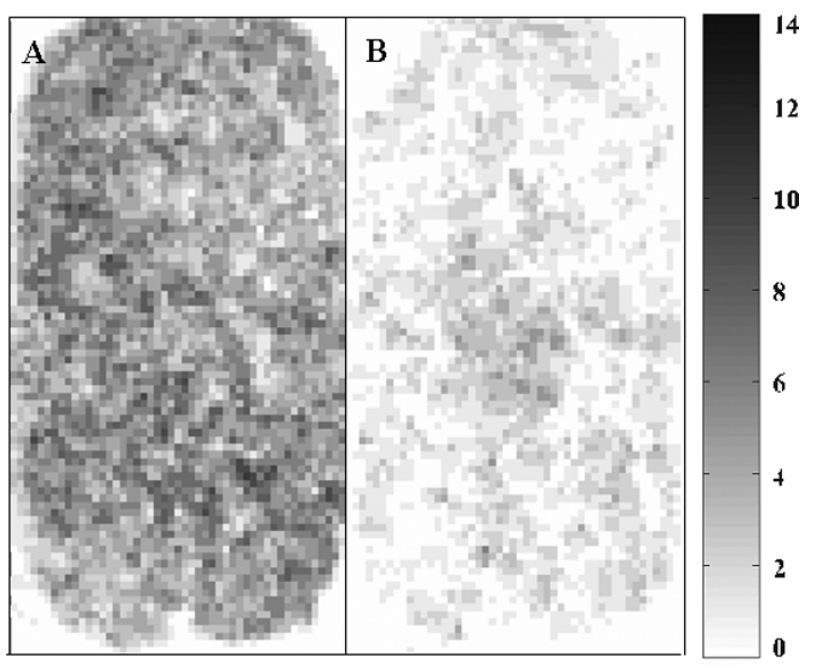

Fig. 6. Histogram of the burns. 14 anodic (B) and 14 cathodic (A) burn patterns were separately summated and represented. The color scale $(0-14)$ indicates the number of occurrences of the detected burns for each individual pixel across the skin surface below the stimulation electrodes.

pare the $\mathrm{CD}$ results to photographic images taken from the skin surface, rather than to histological techniques, because of the ease and convenience of photographic images in documenting the locations of the burns.

Visual examination of the skin surface prior to running the experiments does not suggest obvious locations where burns may occur. The blisters and gross morphological changes occur randomly and cannot be predicted as resulting from Fig. 6 and in the individual experiments [Fig. 3(D)].

The comparison of the pictures taken from the skin after the onset of the burns with CD images shows a clear correlation between the position of the burns and the high current regions. In Fig. 3(D)-(F), we marked some of these locations both on the photo and on the CD images. The measurement is sensitive enough to show regions of high current densities in the pre-burn skin. These correlate with the areas where burn welts were induced and, thus, predict regions were burns are likely to occur. CDI pre-Photo post-(Fig. 5) is not statistically different from CDI post-Photo post $(p=0.92)$. This shows that while CDI pre-predicts regions of high $\mathrm{CD}$, these are confirmed by CDI post. CDI post-CDI pre correlation confirms these predictions. The one-way ANOVA test showed statistically significant differences $(\mathrm{p}<0.05)$ between CDI post-Photo post and CDI post-Photo pre correlation coefficients $(0.02 \pm 0.11)$, respectively between the CDI pre-Photo post-and CDI pre-Photo pre $(r=0.0 \pm 0.12)$. However there is no statistical significant difference between CDI post-Photo pre-and CDI pre-Photo pre $(\mathrm{p}=0.99)$ indicating that the pre-burn photo images cannot predict the regions with increased current flow.

The comparison of CD images with photos raises a couple of issues that influence the values of the correlation coefficients. First of all photos record the aspect of the skin surface as compared to $\mathrm{CD}$ images measuring an averaged value of the current flow within the voxels volume over a slice thickness of $2 \mathrm{~mm}$. Furthermore, the color changes of the skin surface do not necessarily correlate with the intensity of the burn. The appearance of the traumatized areas differs for anodic and cathodic burns [2], and even different coloration could be observed on the same sample. Finally, the photo rescaling process and optical distortions of the camera as well as alignment errors affected the results.

The CDI technique is sensitive to artefacts, which have been discussed elsewhere [32]. Susceptibility artefacts due to the copper lead wire [Fig. 3(B)] that distort the phase image and, thus, the computed $C D$ value, high phase gradients at the current injection site [Fig. 3(A)] and image registration [29], sum up, generating a loss of signal and high positive-negative current transitions as observed in the central region of the $\mathrm{CD}$ images [Fig. 3(E) and (F)]. These artefacts corrupt the local CD values and have, due to different imaging currents levels slightly different aspect in the post-and pre-images. The artefacts are localized and relatively easy to detect. Since the source of these artefacts is generally understood it is reasonable to expect them to be reduced by further development of the CDI technique.

In terms of the numeric values, the correlation coefficient CDI post-Photo post-(0.31 \pm 0.17$)$ and CDI pre-Photo post-(026 \pm $0.16)$ are lower than CDI post-CDI pre-ones $(0.49 \pm 0.17)$ In the last case we encounter only LFCDI specific artefacts where else first two values are affected by all mentioned factors. This explains why the apparently better visual correlations between burn photos and pre, respective post-CDIs are numerically lower than the pre-post-CDI ones.

Based on multiple regression and variance analysis we investigated the differences between the anodic and cathodic burns and the factors that induce them. Taking into consideration both the electrochemical mechanism [2], [8], [16], [49] and the thermal generation hypothesis [6], [20], we investigated charge $(\mathrm{Q})$, current intensity $(\mathrm{I})$, time $(\mathrm{t})$, power $\left(\mathrm{I}^{2}\right)$, and energy $\left(\mathrm{I}^{2} \mathrm{t}\right)$ as possible driving factors. The results summarized in Table I suggest that charge is responsible for low-voltage skin burns. Both in the anodic and cathodic experiments, it accounts for the highest proportion of variability due to the regression $\left(\mathrm{R}^{2} \mathrm{a}\right)$ and provides good statistical significance. There was no evidence supporting the thermal origin, as we have reported in previous work [50] based on in vitro experiments. Our findings support the chemical hypothesis, $\mathrm{pH}$ changes being observed and measured (unpublished data). Extrapolation of the results to human skin should be done with care because of possible morphological differences between porcine and human skin. The linear regression of burn area versus injected charge, for both anodic and cathodic cases shows (Fig. 3) a higher residual variability in the cathodic data set. This can be explained by the mechanism of liquefaction necrosis, [51] which is difficult to control and stop, where else the coagulation necrosis [51] encountered at the anode auto limits the extent of the injury. These two processes also explain the difference between the two slopes, cathodic burns spreading faster than the anodic.

The investigation using LFCDI revealed some interesting findings regarding differences between anodic and cathodic burns due to different underlying mechanism. Acids produce coagulation necrosis by denaturing proteins upon tissue contact. This layer limits further extension of the injury. Alkalis induce a liquefaction necrosis, by denaturing proteins and saponifying fat. There is no limiting mechanism for this, so once initiated, the burn penetrates deeply into the tissue, affecting larger skin area [51]. Because of the lack of a limiting region, burns produced by 


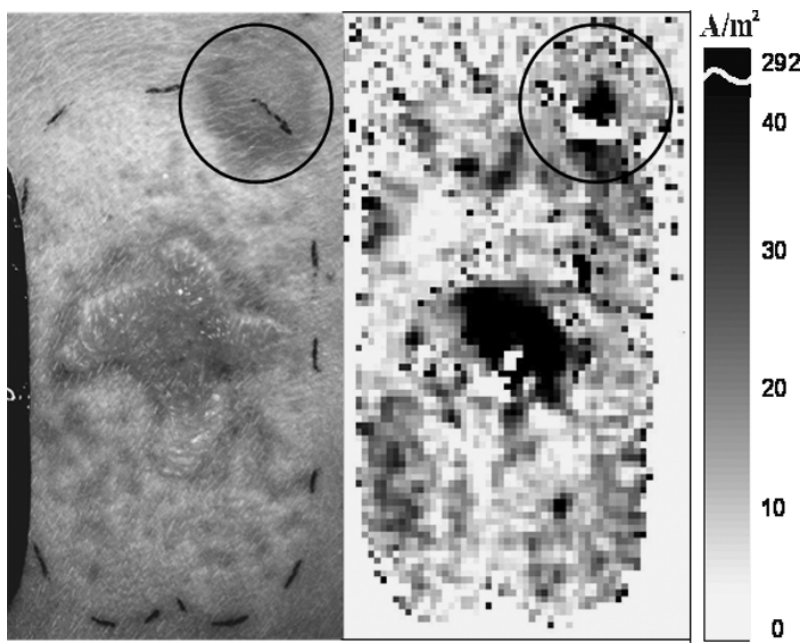

Fig. 7. Photograph of the skin after burn inducing experiment (left). Dotted line reveals the electrode periphery, while the circle locates a burn region. Same area visualized in terms of $\mathrm{CD}$ (image right). Observe the correlation between burns shown in the photo and regions of increased CD. In the center of the circle, a region of low $\mathrm{CD}$ probably identifies a gas bubble developing with the progress of the burn induction process. The amplitude of the imaging current pulses is $50 \mathrm{~mA}$.

alkalis can extend outside the coverage area of the applied surface electrode, inducing current pathways detected by CDI. Fig. 7 illustrates such an example, where the highlighted alkali necrotic area correlates well to the increased CD region. We could observe these morphological changes of the porcine integument outside the area covered by the electrodes in several experiments, but only as a result of cathodic stimulation.

\section{CONCLUSION}

Based on an excised porcine skin-gel model mimicking the skin and underlying soft tissue, we were able to visualize the CD distribution under surface electrodes after burn inducing stimulation. The regions of increased current correlate well with gross morphological changes observed, confirming that LFCDI is a powerful tool in analysing the distribution of current densities in soft tissue. More than that, the method is sensitive enough to detect and determine regions of low impedance in the skin, predicting the current distribution even before the burns occur. Visual analysis of the induced burns has revealed a rather uniform distribution of the cathodic burns with a slight increase corresponding to the current injection site in the anodic case. This is contradicting previous observations [2] concerning the edge effect, and we consider the differences arising from the type of electrodes used. Both types of resulting burns seem to be charge dependent, and there is no statistical evidence that thermal mechanisms could be of origin. A better understanding of the current path trough the skin, as well as optimized electrode design could lead to a more uniform CD distribution during electrical stimulation and, hence, decrease the incidence of reported skin damages.

\section{ACKNOWLEDGMENT}

The authors wish to thank J.C. Mortensen, for his help with the porcine skin, F.T. Jensen for the MRI support and AMBU A/S for providing the electrodes.

\section{REFERENCES}

[1] R. B. Giffin Jr. and C. Susskind, "EEG skin "burn": electrical or chemical?," Med. Res. Eng, vol. 6, no. 4, pp. 32-34, 1967.

[2] M. N. Leeming, C. Ray, and W. S. Howland, "Low-voltage, direct-current burns," J. Am. Med. Assoc., vol. 214, no. 9, pp. 1681-1684, 1970.

[3] M. N. Leeming, R. G. Jacobs, and W. S. Howland, "Low-voltage, direct-current plethysmograph burns," Med. Res. Eng., vol. 10, no. 5, pp. 19-21, 1971.

[4] C. W. Jarvis and D. A. Voita, "Low voltage skin burns," Pediatrics, vol. 48, no. 5, pp. 831-832, 1971.

[5] A. Etchin and Y. Mamet, "Electrochemical skin burn after trans-cutaneous electronerve analgesia," Anesth. Analgesia, vol. 61, no. 9, pp. 801-802, 1982

[6] M. T. Balmaseda, M. T. Fatehi, S. H. Koozekanani, and J. S. Sheppard, "Burns in functional electric-stimulation-2 case-reports," Arch. Physical Med. Rehabil., vol. 68, no. 7, pp. 452-453, Jul. 1987.

[7] J. B. Cooper, R. Decesare, and M. N. Dambra, "An engineering critical incident-direct-current burn from a neuromuscular stimulator," Anesthesiology, vol. 73, no. 1, pp. 168-172, Jul 1990.

[8] P. Borrie and J. C. B. Fenton, "Buzzer ulcers," Br. Med. J., vol. 2, no. 5506, pp. 151-152, 1966.

[9] M. W. Greaves, "Scarring due to enuresis blankets," Br. J. Dermatol., vol. 81, no. 6, pp. 440-442, 1969.

[10] M. W. Hursthouse, "Burns from enuresis alarm apparatus-case report," New Zealand Med. J., vol. 79, no. 499, pp. 258-259, 1973.

[11] M. Lippmann and W. A. Fields, "Burns of Skin Caused by A Peripheral Nerve Stimulator," Anesthesiology, vol. 40, no. 1, pp. 82-84, 1974.

[12] E. A. Grossi, M. A. Parish, M. R. Kralik, L. R. Glassman, R. A. Esposito, G. H. Ribakove, A. C. Galloway, and S. B. Colvin, "Direct-current injury from external pacemaker results in tissue electrolysis," Ann. Thoracic Surg., vol. 56, no. 1, pp. 156-157, Jul. 1993.

[13] A. Haenggeli, M. Richter, W. Lehmann, and P. Dulguerov, "A complication of intraoperative facial nerve monitoring: facial skin burns," Am. J. Otol., vol. 20, no. 5, pp. 679-682, Sep.. 1999.

[14] T. Lewis and Y. Zottermann, "Vascular reactions of the skin to injury, part VIII: the resistence of the human skin to constant curents, in relation to injury and vascular response," J. Physiol., vol. 62, pp. 280-288, 1927.

[15] Klinische Wochenschrift, vol. 16, no. 90, p. 93, 1937.

[16] H. Molitor and L. Fernandez, "Studies on iontophoresis. I. Experimental studies on the causes and prevention of iontophoretic burns," Am. J. Med. Sci., vol. 198, pp. 778-785, 1939.

[17] C. F. Mandleco, "Application of Iontophoresis for Noninvasive Administration of Lidocaine Hydrocloride in the Ionized Form," Univ. Utah, Salt Lake City, 1978.

[18] J. S. Guffey, M. J. Rutherford, W. Payne, and C. Phillips, "Skin pH changes associated with iontophoresis," J. Orthop. Sports Phys. Therapy, vol. 29, no. 11, pp. 655-660, Nov. 1999.

[19] M. R. Prausnitz, "The effects of electric current applied to skin: a review for transdermal drug delivery," Adv. Drug Delivery Rev., vol. 18, no. 3, pp. 395-425, Feb. 1996.

[20] H. Lambert, E. DeBaetselier, G. Vanalme, and G. De Mey, "Skin burn risks using transcutaneous direct current," in Proc. IEEE Eng. Med. Biol. 17th Аnпи. Conf., Sep. 1995, pp. 477-478.

[21] R. R. Burnette and B. Ongpipattanakul, "Characterization of the pore transport properties and tissue alteration of excised human skin during iontophoresis," J. Pharm. Sci., vol. 77, no. 2, pp. 132-137, Feb. 1988.

[22] C. Cullander and R. H. Guy, "Sites of iontophoretic current flow into the skin: identification and characterization with the vibrating probe electrode," J. Invest. Dermatol., vol. 97, no. 1, pp. 55-64, Jul. 1991.

[23] O. G. Martinsen, S. Grimnes, L. Morkrid, and M. Hareide, "Line patterns in the mosaic electrical properties of human skin-a cross-correlation study," IEEE Trans. Biomed. Eng, vol. 48, no. 6, pp. 731-734, Jun. 2001.

[24] S. Grimnes, "Pathways of ionic flow through human skin in vivo," Acta Dermatol. Venereol., vol. 64, no. 2, pp. 93-98, 1984.

[25] E. R. Scott, A. I. Laplaza, H. S. White, and J. B. Phipps, "Transport of ionic species in skin: contribution of pores to the overall skin conductance," Pharm. Res., vol. 10, no. 12, pp. 1699-1709, Dec. 1993.

[26] M. R. Prausnitz, "Imaging regions of transport across human stratum corneum during high-voltage and low-voltage exposures," J. Pharmaceutical Sci., vol. 85, no. 12, pp. 1363-1370, 1996.

[27] H. A. Abramson and M. H. Gorin, "Skin reactions. IX: The electrophoretic demonstration of the patent pores of the living human skin," J. Phys. Chem., vol. 44, pp. 1094-1102, 1940.

[28] M. L. Joy, G. Scott, and M. Henkelman, "In vivo detection of applied electric currents by magnetic resonance imaging," Magn. Reson. Imag., vol. 7, no. 1, pp. 89-94, Jan. 1989.

[29] M. L. Joy, V. P. Lebedev, and J. S. Gati, "Imaging of current density and current pathways in rabbit brain during transcranial electrostimulation," IEEE Trans. Biomed. Eng, vol. 46, no. 9, pp. 1139-1149, Sep. 1999.

[30] R. S. Yoon, T. P. DeMonte, K. F. Hasanov, D. B. Jorgenson, and M. L. G. Joy, "Measurement of thoracic current flow in pigs for the study of defibrillation and cardioversion," IEEE Trans. Biomed. Eng., vol. 50, no. 10 , pp. $1167-1173$, Oct. 2003. 
[31] G. C. Scott, M. L. G. Joy, R. L. Armstrong, and R. M. Henkelman, "Measurement of nonuniform current-density by magnetic-resonance," IEEE Trans. Med. Imag., vol. 10, no. 3, pp. 362-374, Sep. 1991.

[32] — , "Sensitivity of magnetic-resonance current-density imaging," $J$. Magn. Reson., vol. 97, no. 2, pp. 235-254, Apr. 1992.

[33] A. Patriciu, K. Yoshida, T. P. DeMonte, and M. L. G. Joy, "Detecting skin burns induced by surface electrodes," in Proc. 23rd Annu. Int. Conf. IEEE Engineering in Medicine and Biology Society, vol. 3, 2001, pp. 3129-3131.

[34] W. Montagna and J. S. Yun, "The skin of the domestic pig," J. Invest. Dermatol., vol. 42, pp. 11-21, Jul. 1964.

[35] N. A. Monteiro-Riviere, Swine in Biomedical Research, M. E. Tubleson, Ed. New York: Plenum, 1986, pp. 641-655.

[36] N. A. Monteiro-Riviere and M. W. Stromberg, "Ultrastructure of the integument of the domestic pig (Sus scrofa) from one through fourteen weeks of age," Anat. Histol. Embryol., vol. 14, no. 2, pp. 97-115, Jun. 1985.

[37] M. J. Bartek, J. A. LaBudde, and H. I. Maibach, "Skin permeability in vivo: comparison in rat, rabbit, pig and man," J. Invest. Dermatol., vol. 58, no. 3, pp. 114-123, Mar. 1972.

[38] W. G. Reifenrath, E. M. Chellquist, E. A. Shipwash, and W. W. Jederberg, "Evaluation of animal models for predicting skin penetration in man," Fundam. Appl. Toxicol., vol. 4, no. 2, pp. S224-S230, Apr. 1984.

[39] D. Rouge, A. Polynice, J. L. Grolleau, B. Nicoulet, J. P. Chavoin, and M. Costagliola, "Histologic assessment of low-voltage electrical burns: experimental study with pigskin," J. Burn Care Rehabil., vol. 15, no. 4, pp. 328-334, Jul. 1994.

[40] L. C. Laberge, P. A. Ballard, and R. K. Daniel, "Experimental electrical burns: low voltage," Ann. Plast. Surg., vol. 13, no. 3, pp. 185-190, Sep. 1984

[41] J. R. Stanley, O. M. Alvarez, E. W. Bere, W. H. Eaglstein, and S. I. Katz, "Detection of basement-membrane zone antigens during epidermal wound-healing," Clin. Res., vol. 29, no. 2, p. A614, 1981.

[42] L. Danielsen, I. K. Genefke, T. Karlsmark, S. Lorenzen, K. G. Nielsen, O. Nielsen, H. K. Thomsen, and O. Aalund, "[Thermal and electric skin injuries in swine]," Ugeskr. Laeger, vol. 140, no. 51, pp. 3191-3197, Dec. 1978.

[43] H. K. Thomsen, L. Danielsen, O. Nielsen, O. Aalund, K. G. Nielsen, T. Karlsmark, and I. K. Genefke, "Early epidermal changes in heat- and electrically injured pig skin. I. A light microscopic study," Forensic Sci. Int., vol. 17, no. 2, pp. 133-143, Mar. 1981.

[44] J. C. Blechinger, E. L. Madsen, and G. R. Frank, "Tissue-mimicking gelatin-agar gels for use in magnetic resonance imaging phantoms," Med. Phys., vol. 15, no. 4, pp. 629-636, Jul. 1988.

[45] G. Yip, M. L. G. Joy, G. C. Scott, and R. M. Henkelman, "In vivo current density imaging," in Proc. 11th Annu. Meeting, vol. 2, Berlin, Germany, 1992 , p. 3917.

[46] P. W. Ledger, "Skin biological issues in electrically enhanced transdermal delivery," Adv. Drug Delivery Reviews, vol. 9, no. 2-3, pp. 289-307, Sep. 1992.

[47] A. K. Banga and Y. W. Chien, "Iontophoretic delivery of drugs-fundamentals, developments and biomedical applications," J. Controlled Release, vol. 7, no. 1, pp. 1-24, Apr. 1988.

[48] P. Armitage and G. Berry, Statistical Methods in Medical Research, 3rd ed. Oxford, U.K.: Blackwell Science, 1998.

[49] C. T. Costello and A. H. Jeske, "Iontophoresis-applications in transdermal medication delivery," Phys. Therapy, vol. 75, no. 6, pp. 554-563, Jun. 1995

[50] K. Yoshida and A. Patriciu, "Detecting skin burns induced by surface electrodes," in Proc. 5th Annu. Conf. Int. Functional Electrical Stimulation Society, Jun. 2000, pp. 282-284.

[51] A. G. Garza, "Thermal and chemical burns," in Just the Facts in Emergency Medicine, D. M. Cline, O. John Ma, and J. E. Tintinalli, Eds. New York: McGraw Hill, 2000, pp. 377-380.

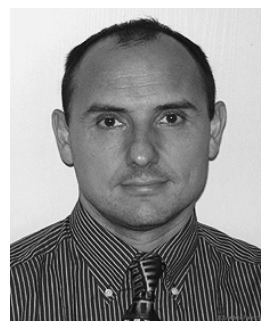

Andrei Patriciu ( $\mathrm{S}^{\prime}$ 99) was born in Cluj-Napoca, Romania. He received the M.Sc.E.E. degree from the Technical University, Cluj-Napoca, Romania, in 1986 . He is currently working toward the Ph.D degree in biomedical engineering at the Center for Sensory Motor-Interaction, Aalborg University, Denmark.

From 1986-1998, he worked as a Researcher at the Babes-Bolyai University and the Bitnet Research Center in Cluj-Napoca. His research interests include surface electrical stimulation and surface stimulation electrode design and optimization.

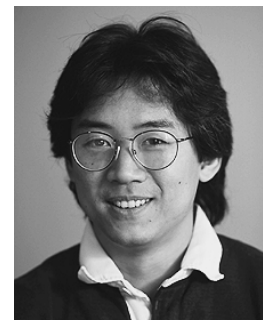

Ken Yoshida (S'89-M'91) was born in Los Angeles, CA, in 1965. He received the B.S.E. degree in bioengineering in 1989 from the University of California at Los Angeles, and the Ph.D. degree in bioengineering from the University of Utah, Salt Lake City, in 1994.

From 1995 to 1998, he was an Alberta Heritage Foundation for Medical Research and a Canadian NeuroScience Network Postdoctoral Fellow at the University of Alberta, Edmonton, AB, Canada, in the Division of Neuroscience. He is currently an Associate Professor in the Department of Health Science and Technology and the Center for Sensory-Motor Interaction at Aalborg University, Aalborg, Denmark. His research focus is the development of selective neural interfaces, and the application of these devices to study natural neuromuscular control and to investigate natural sensor based FNS systems.

Dr. Yoshida is a member of the Society for Neuroscience, the International Functional Electrical Stimulation Society, the Biomedical Engineering Society, and Tau Beta Pi.

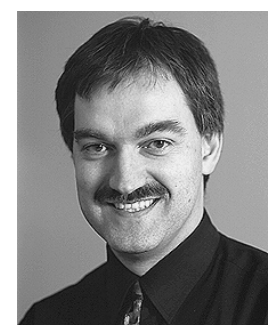

Johannes J. Struijk was born in Rijssen, The Netherlands, in 1963. He received the M.Sc. degree in electrical and biomedical engineering and the $\mathrm{Ph} . \mathrm{D}$. degree in electrical engineering from the University of Twente, Enschede, The Netherlands, in 1988 and 1992, respectively.

After two years as a Postdoctoral Fellow at the Institute of Biomedical Technology, University of Twente, he joined the Center for Sensory-Motor Interaction and the Department of Health Science and Technology at Aalborg University, Aalborg, Denmark, where he currently is Associate Professor and Director of Studies for programs in Biomedical Engineering and Informatics. His research interests are in the fields of neuroprostheses, bioelectricity, and cardiology.

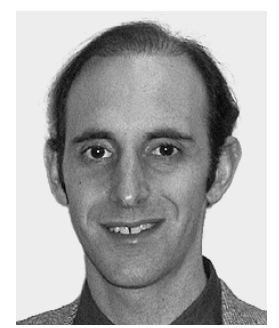

Tim P. DeMonte (M'91) was born in Canada in 1972. He received the B.A.Sc. degree in electrical engineering from the University of Windsor, Windsor, ON, Canada, in 1995. He received the M.A.Sc. degree in a collaborative program that includes electrical and biomedical engineering from the University of Toronto, Toronto, ON, Canada, in 2001.

Presently, he is working under contract with the University of Toronto to perform research related to defibrillation, cardiac imaging, current density imaging, and impedance imaging. His research interests include imaging of electrical quantities in biological systems.

Mr. DeMonte is a member of Sigma Xi, The Scientific Research Society.

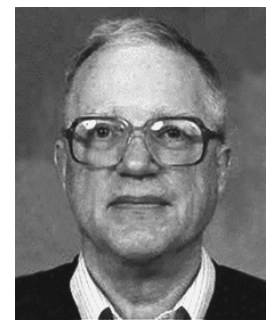

Michael L. G. Joy (M'83) received the B.Sc. degree in physics and the Ph.D. degree in electrical engineering from the University of Toronto, Toronto, ON, Canada, in 1963 and 1970, respectively.

Since 1970, he has been cross appointed between what is now the Institute of Biomaterials and Biomedical Engineering where he is the Associate Director (Graduate Studies) and the Department of Electrical and Computer Engineering where he recently served a term as the Associate Chair for the Undergraduate Programs. His research has always been in the field of medical imaging and has ranged from building gamma ray cameras and coded aperture imaging to magnetic resonance imaging. His goal has been to develop new imaging modalities.

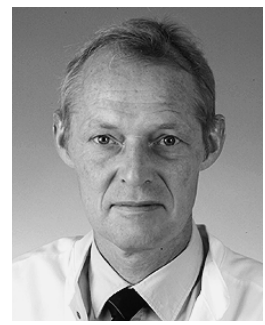

Hans Stødkilde-Jørgensen is Head of the MR Research Centre at Skejby University Hospital. Through nine years chairman of the Danish Society of Biomedical Engineering. Board member of the Alexandra Institute (the Institute for IT of Denmark). He has more than 100 journal publications and book chapters. $\mathrm{He}$ is especially interested in functional and neuro studies and oncology. 